With saponin solution, it was found possible to form rather large floating drops (11 $\mathrm{mm}$. in diameter) by successively dropping on single drops. The foreign 'adsorbed' layer necessary may also be realized by using a thin layer of oil, spread on a water surface. When dropping on water, very large floating drops may be produced, as evidenced in Fig. 3, showing drops formed by the coalescence : in $a$ of 30 single drops, in $b$ of about 80 drops (diameter $16 \mathrm{~mm}$.). These fairly stable spherically limited masses of water in water with an extremely thin oil layer remind one of the spherical oil masses in alcohol of the same density produced by Plateau.

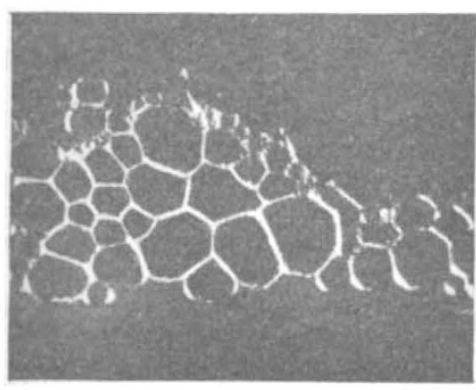

Fig. 4.

We were further led to the production of a foam, consisting solely of liquids. Such a 'liquid-foam' is evidenced by Fig. 4. The cell walls are saponin solution $(0.5$ per cent), the cell content is oil ('Lighthouse', Pratt), which has been introduced into the saponin solution-kept at the bottom of a thin cuvette.

The appearance of the fluid-foam was very similar to the 'gas-foam' formed in the same cuvette on introducing air into the saponin solution.

The main interest of the fluid-foam is that it affords a rather close analogy with the capillary grain structure of solid metals ${ }^{2}$.

A detailed account of this work will appear in Arkiv för Matematik, Astronomi och Fysik, vol. $30 \mathrm{~A}$, of the Royal Swedish Academy of Sciences, Stockholm.

Laboratorium C. Benedicks,

Cart Benedicks. Per Sederholm.

Metallografiska Institutet, Stockholm.

${ }^{1}$ Hazlehurst and Neville, J. Phys. Chem., 41, 1205 (1937).

${ }^{2}$ Cf. Benedicks, 180. Congrès de Chimie Indust. (Nancy, 1938). Chimie et Industrie (Paris, 1939). Koll. Z., 91, 217 (1940).

\section{Interpretation of Patterson Diagrams}

Prof. J. Monteath Rober'sson's communication on the above subject in NATURE ${ }^{1}$ suggests a modified method, based on the same geometrical principle.

I refer now to the diagram in Prof. Robertson's communication. Let $A A^{\prime}$ and $B B^{\prime}$ be opaque screens pierced in the points $1,2, \ldots$ and $1^{\prime}, 2^{\prime}, \ldots$ respectively. If $A A^{\prime}$ is illuminated from the left, the peaks in the Patterson diagram will appear on the surface $C C^{\prime}$.

The two screens $A A^{\prime}$ and $B B^{\prime}$ can be rapidly prepared by punching circular holes in sheets of cardboard. The holes form the pattern of the projected atomic positions and the pattern on $A A^{\prime}$ may be drawn to a scale twice as large as that of the pattern on $B B^{\prime}$. In this case the distance from $A A^{\prime}$ to $B B^{\prime}$ should be equal to the distance from $B B^{\prime}$ to $C C^{\prime}$. The unit area will then be the same on $A A^{\prime}$ as on $C C^{\prime}$. The integrated intensity of a projected Patterson peak will be proportional to the product of the areas of the two corresponding holes. If the distances between the screens are large compared with the extension of the atomic patterns, and if the holes in $A A^{\prime}$ and $B B^{\prime}$ are made to correspond to atomic contours, the contours of the Patterson peaks will be practically circular and the diameter of a circle will be the sum of the diameters of the two corresponding atom holes in $A A^{\prime}$. Important features of the real Patterson function are thus rather closely attained.

The projection surface $C C$ is preferably a groundglass plate, which is viewed from the right. The contours of the real Patterson diagram can be drawn on the ground-glass surface, thus enabling an easy comparison with the projected picture.

Compared with Prof. Robertson's method, the modified method outlined here lacks the possibility of continuous variation. However, the variation can be carried out quite rapidly, and the modified method seems to possess certain advantages in other respects.

Chemical Institute of the University, G. HÄGG.

$$
\begin{aligned}
& \text { Uppsala. } \\
& \text { Nov. } 3 .
\end{aligned}
$$

${ }^{1}$ Nature, 152, 411 (1943)

\section{A New Type of Microphotometer}

UNDER this title ${ }^{1} R$. Fürth gave a short description of an instrument designed for providing a practically instantaneous record on a cathode ray oscillograph of the density distribution along a short straight line on a photographic plate. This record was in fact obtained by the superposition of two traces writing in opposite directions on the screen of the cathode ray oscillograph, corresponding to the two directions of the vibrational motion of the plate through the slit image. A rather thick curve resulted, therefore, with loss in detail compared with the records of other existing types of microphotometers. In addition, fluctuations in frequency of the mains voltage by which the instrument is operated could cause slight displacements of the two traces ${ }^{2}$.

A brief description is given here of an improvement on Fürth's microphotometer which has resulted in an increased resolving power and more satisfactory working conditions. It consists in the application between grid and cathode of the cathode ray tube of a periodic 'square wave' voltage which controls the brightness of the fluorescent spot on the screen in such a way as to make only one trace visible. To produce this voltage (which must be properly related to the time-base voltage) an electrical circuit is used which is comprised mainly of a $90^{\circ}$ phase-shifting network with an amplifier the output of which is applied to a peak-clipping device.

In order to demonstrate the improved performance of the instrument, three photographic records are shown relating to the density distribution in a photograph of the arc spectrum of copper in the neighbourhood of 3440 A., taken with an ordinary glass spectrograph. Records 2 and 3 (with original magnification of 30 ) each cover a range of $15 \mathrm{~A}$. approximately. Record $\mathrm{I}$ is of the central hump in record 2; it corresponds to an original magnification of 200 and covers a range of $2 \cdot 2 \mathrm{~A}$., and a distance of $0.24 \mathrm{~mm}$. 